\title{
ISOLATION OF 8-HIDROXY-6,7-DIMETHOXY COUMARIN FROM JARAK TINTIR STEM (Jatropha multifida L.) AND ITS TOXICITY VALUE USING BRINE SHRIMP LETHALITY TEST (BSLT)
}

\author{
ISOLASI DARI 8-HIDROKSI-6,7-DIMETHOKSI KUMARIN DAN TOKSISITAS BATANG \\ JARAK TINTIR (Jatropha multifida L.) MENGGUNAKAN BRINE SHRIMP LETHALITY \\ TEST (BSLT)
}

\author{
Akhirul Kahfi Syam ${ }^{1,2 *}$, Muhamad Insanu², Komar Ruslan Wirasutisna ${ }^{2}$ \\ 1 Jenderal Achmad Yani University Cimahi-Indonesia \\ ${ }^{2}$ School of Pharmacy Institut Teknologi Bandung-Indonesia
}

\begin{abstract}
Jatropha multifida L. (jarak tintir) was a shrub, annual, and had $\pm 2 \mathrm{~m}$ high. Empirically jarak tintir sap was used as traditional medicine by Indonesian people for a long time. Only limited studies were conducted regarding its chemical compound. It was reported that multifidone (diterpenoid compound from the stem) had an activity against cancer cells in vitro. This study aimed to test the toxicity of various extracts (n-hexane, ethyl acetate, and methanol) with Brine Shrimp Lethality Test (BSLT) of Jatropha multifida L. stem. A Fraxidin (8-hidroxy-6,7-dimethoxy coumarin) has been isolated from ethyl acetate fraction based on highest cytotoxic with $L C_{50}$ value $3.69 \mu \mathrm{g} / \mathrm{mL}$. The isolated compound was elucidated to gain chemical structure base on spectroscopic data (UV-Vis Spectrofotometric, IR Spectrofotometric, and NMR). Toxicity of fraxidin was tested on BSLT and showed no potential activity with $L C_{50}$ value $>500 \mu \mathrm{g} / \mathrm{mL}$.
\end{abstract}

Key words: Fraxidin, BSLT, coumarin, Jatropha multifida L.

\begin{abstract}
ABSTRAK
Jatropha multifida L. (jarak tintir) adalah semak yang memiliki tinggi $\pm 2 \mathrm{~m}$. Secara empiris getah Jarak tintir digunakan sebagai obat tradisional oleh penduduk Indonesia sejak lama. Penelitian yang dilakukan tentang senyawa kimia Jarak tintir masih terbatas. Menurut penelitian, multifidone (senyawa diterpenoid dari batang Jarak tintir) memiliki aktivitas terhadap sel kanker in vitro. Penelitian ini bertujuan untuk mengetahui toksisitas berbagai macam ekstrak (n-hexane, etil asetat, dan metanol) dari batang Jatropha multifida L. dengan Brine Shrimp Lethality Test (BSLT). Sebuah Fraksidin (8-hidroksi-6,7-dimetoksi kumarin) telah diisolasi dari fraksi etil asetat berdasarkan sitotoksik tertinggi dengan nilai $L C_{50} 3.69 \mu \mathrm{g} / \mathrm{mL}$. Senyawa yang diisolasi diuraikan untuk mendapatkan basis struktur kimia pada data spektroskopik (UV-Vis Spektrofotometrik, IR Spektrofotometrik, dan NMR). Toksisitas fraksidin diuji dengan BSLT dan tidak menunjukkan potensi aktivitas dengan nilai $L C_{50}>500 \mu \mathrm{g} / \mathrm{mL}$.
\end{abstract}

Kata kunci: Fraksidin, BSLT, kumarin, Jatropha multifida L.

\section{INTRODUCTION}

Jatropha multifida L. (jarak tintir) is a shrub, annual, and had $\pm 2 \mathrm{~m}$ high. Empirically Jarak tintir sap has been used as a traditional medicine by Indonesian people for a long time (Hutapea, 2000). It was reported that multifidone (diterpenoid compound from the stem) has an activity against cancer cells in vitro with $\mathrm{IC}_{50}$ value was 45-160 $\mu \mathrm{M}$ (Das et al., 2008b). Another ditepenoid compound from the stem had also been found, such as 15-0-acetyl japodagrone, (4E)-jatrogrossidenta-dione (Das et al., 2008a),

Corresponding Author: Akhirul Kahfi Syam

Email: akhirulkahfisyam@yahoo.co.id multidione (Das et al., 2009), multifidanol and multifidenol (Kanth et al., 2011). Cytotoxic assay of Jarak tintir leaves has been done and given $\mathrm{LC}_{50}$ value $0,91 \mu \mathrm{g} / \mathrm{mL}$ of $n$-hexane fraction (Honasan, 2012).

Brine shrimp lethality test (BSLT) was one of toxicity assays that used toxic bioactive study from natural products. This metode is known as bioassay-guided fractionation from natural products, because it is easy, fast, cheap, and good enough in reproducible (Meyer et al., 1982). This study reports on the isolation, toxicity test, and structure elucidation of fraxidin (8-hidroxy6,7-dimethoxy coumarin) from ethyl acetate fraction. 


\section{MATERIAL AND METHODS \\ Sample Preparation}

J. multifida stem is collected from Manoco garden, Lembang, West Java-Indonesia, and determined in Herbarium Bandungese School of Life Science and Technology, ITB. The stem was dried under morning sunlight. The dried stems were grinded until a crude drug powder was obtained.

\section{Characterization of Crude Drug}

The crude drug was examined microscopically and characterized such as moisture, total ash, water soluble ash, acid insoluble ash, water soluble extract, and ethanol soluble extract content (Depkes, 2000).

\section{Phytochemical Screening of Crude Drug and Extract}

Phytochemical screening was done to detect secondary metabolite, like alkaloids, flavonoids, polyphenols, tannins, quinones, saponins, monoterpenoids-sesquiterpenoids and terpenoids-triterpenoids (Farnsworth, 1966).

\section{Extraction}

Continous extraction was performed with Soxhlet apparatus using three solvents with increasing polarity (n-hexane, ethyl acetate, and methanol).

\section{Brine Shrimp Lethality Test (BSLT)}

All extracts were tested using BSLT toxicity assay with various concentrations. Lethal concentration $50 \% \quad\left(\mathrm{LC}_{50}\right)$ was counted and obtained from Probit analysis. The lowest $\mathrm{LC}_{50}$ of extract was the most toxic and has potential activity in cytotoxic (Meyer et al., 1982).

\section{Isolation}

Ethyl acetate fraction was fractionated using vacuum liquid chromatography (VLC) with gradien elution (dichloromethane-ethyl acetatemethanol). Fraction $6 \mathrm{~K}, 7 \mathrm{~K}$ and $8 \mathrm{~K}$ were fractionated with VLC with gradien elution (dichloromethane-ethyl acetate-methanol). Sub fractions 8-10 were then decanted by some organic solvents to obtain isolate 1 .

\section{Chemical Compound Characterization and BSLT Assay Isolate 1}

Isolate 1 was characterized using specific spray reagent, melting point assay, UV-Vis, infrared spectrophotometry, and H-NMR, C-NMR and 2D-NMR spectrometry. The toxicity of isolate 1 was tested with BSLT.

\section{RESULT AND DISCUSSION \\ Characterization Crude Drug}

Microscopic examination showed fragments of parenchyma, crystal, and tracheid (Figure 1). The characterization of crude simplicia is water content $3.02 \pm 0.05 \% \mathrm{v} / \mathrm{w}$; total ash content 5.17 $\pm 0.54 \% \mathrm{w} / \mathrm{w}$; water soluble ash content $2.04 \pm$ $0.15 \% \mathrm{w} / \mathrm{w}$; non-soluble acidic ash content $0.36 \pm$ $0.07 \% \mathrm{w} / \mathrm{w}$; water soluble extractable matter $11.46 \pm 0.57 \% \mathrm{w} / \mathrm{w}$; ethanol soluble extractable matter $7.56 \pm 0.33 \% \mathrm{w} / \mathrm{w}$ and all parameters fullfil the regulation quality of crude drug.

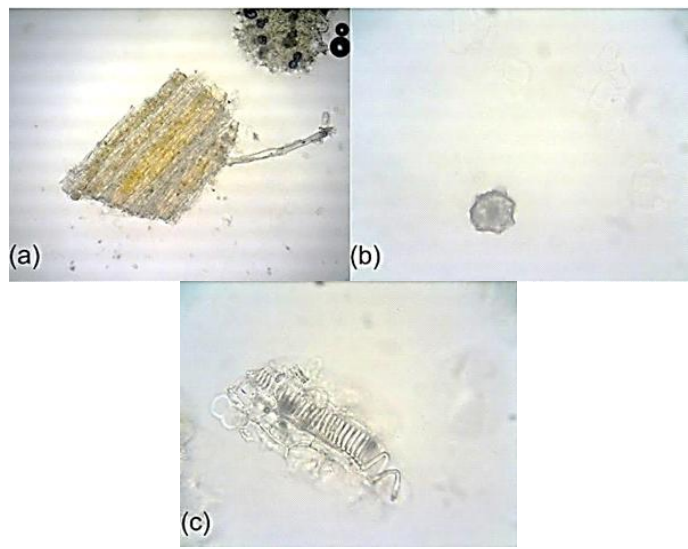

Figure 1. Microscopic examination crude drug of $J$. multifida L. in chloral hidrate, (a) parenchyma (100x), (b) crystal (400x), (c) tracheid (400x).

\section{Brine Shrimp Lethality Test (BSLT)}

Brine shrimp lethality test (Table I) of extract and isolate1 was done between 1-100 $\mu \mathrm{g} / \mathrm{mL}$ againts Artemia salina. Probit analysis showed $\mathrm{LC}_{50}$ of methanol, ethyl acetate, and $\mathrm{n}$ hexane extracts were $4.85 ; 3.69$ and $18.14 \mu \mathrm{g} / \mathrm{mL}$ respectively. Ethyl acetate was the most active and toxic. A compound called as cytotoxic if the compound has $\mathrm{LC}_{50} \leq 30 \mu \mathrm{g} / \mathrm{mL}$ (McLaughlin dan Jerry, 1991).

Table I. Brine Shrimp Lethality Test (BSLT) of extracts and Isolate1

\begin{tabular}{ccccc}
\hline Extract & Methanol & $\begin{array}{c}\text { Ethyl } \\
\text { Acetate }\end{array}$ & $\begin{array}{c}\mathbf{n}- \\
\text { hexane }\end{array}$ & $\begin{array}{c}\text { Isolate } \\
\mathbf{1}\end{array}$ \\
\hline $\begin{array}{c}\mathrm{LC}_{50} \\
(\mathrm{ppm})\end{array}$ & 4.85 & 3.69 & 18.14 & $>500$ \\
\hline
\end{tabular}

\section{Phytochemical Screening}

The crude drug showed only alkaloids and tannin that gave negative result. Ethyl acetate as most active extract had flavonoid, polyphenol, quinon, monoterpen-sesquiterpen, and steroidtriterpenoid for compound that responsible to its activity. 


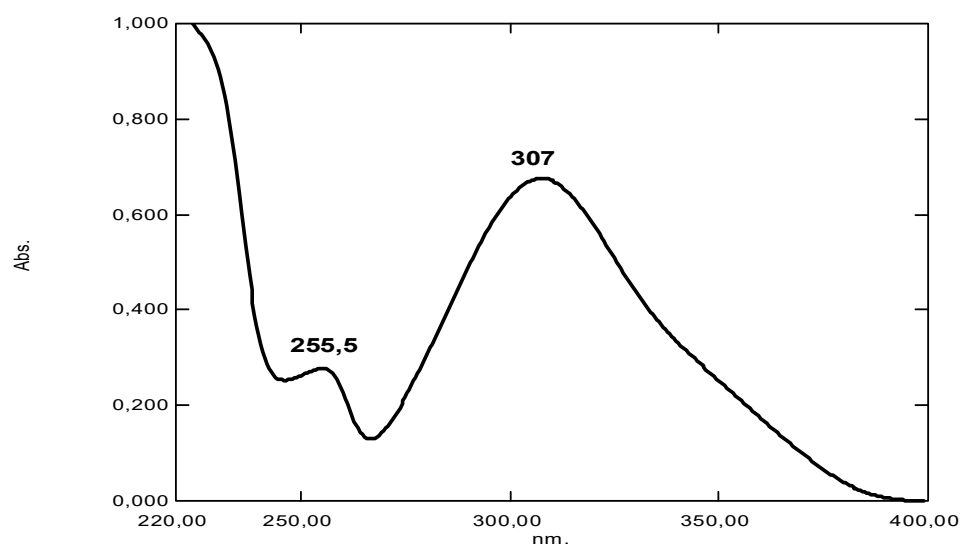

Figure 2. UV spectrum of isolat 1 in methanol

Table II. Phytochemical Screening Crude Drug and Ethyl Acetate Extracts of J. Multifida L.

\begin{tabular}{lccc}
\hline \multicolumn{1}{c}{ Compound } & Reagent & Crude Drug & Extract \\
\hline Alkaloid & Dragendorff & - & - \\
Flavonoid & Zn, amyl alcohol & + & + \\
Polyphenol & $\mathrm{FeCl}_{3}$ & + & + \\
Quinon & $\mathrm{KOH} \mathrm{1 \%}$ & + & + \\
Saponin & & + & - \\
Tannins & Gelatin 1\% & - & - \\
Monoterpen-sesquitepen & Vanilin-SO & + & + \\
Steroid-Triterpenoid & Liebermann Bourchard & + & + \\
\hline
\end{tabular}

Table III. Comparison ${ }^{1} \mathrm{H}-\mathrm{NMR}$ and ${ }^{13} \mathrm{C}$-NMR data of isolate 1 with Fraxidin

\begin{tabular}{|c|c|c|c|c|}
\hline \multirow{2}{*}{ Position } & \multicolumn{2}{|c|}{ Isolate 1 (Aseton-d 6 ) } & \multicolumn{2}{|c|}{ Fraxidin $\left(\mathrm{CDCl}_{3}\right)$} \\
\hline & $\delta_{H}(p p m)(500 \mathrm{MHz})$ & $\delta \mathrm{c}(\mathrm{ppm})(125 \mathrm{MHz})$ & $\delta_{H}(p p m)(400 \mathrm{MHz})$ & $\delta_{\mathrm{c}}(\mathrm{ppm})(100 \mathrm{MHz})$ \\
\hline 2 & - & 160.7039 & - & 161.4 \\
\hline 3 & $6.2911 d$ & 115.5018 & $6.26 d$ & 114.4 \\
\hline 4 & $7.8600 d$ & 144.9752 & $7.60 d$ & 144.4 \\
\hline 5 & $6.7983 s$ & 101.1943 & $6.44 s$ & 99.7 \\
\hline 6 & - & 150.9939 & - & 150.2 \\
\hline $6-\mathrm{OCH}_{3}$ & $3.8773 s$ & 56.5453 & 3.83 & 56.1 \\
\hline 7 & - & 140.9119 & - & 140.4 \\
\hline $7-\mathrm{OCH}_{3}$ & $3.8850 s$ & 61.2191 & 3.91 & 61.0 \\
\hline 8 & - & 139.3095 & - & 138.5 \\
\hline 9 & - & 139.3762 & - & 138.3 \\
\hline 10 & - & 115.7498 & - & 114.1 \\
\hline
\end{tabular}

\section{Chemical Compound Characterization}

Isolate 1 , white solid, $115.1 \mathrm{mg}, \mathrm{mp} 195.8^{\circ}$ $196.8^{\circ} \mathrm{C}$ were obtained using two step vacuum liquid chromatography (VLC) with gradien elution (dichloromethane-ethyl acetate) from ethyl acetate extract. UV-Visible Spectrophotometer: Isolate 1 showed 2 peaks on $\lambda \max 255.5$ and 307 nm (Figure 2.)

IR Spectrophotometer (KBr medium): $3228.84 \quad \mathrm{~cm}^{-1}(-\mathrm{OH}) ; \quad 3055.24 ; 3016.67 \mathrm{~cm}^{-1}$ (aromatic C-H); 2989.66; 2947.23; $2839.22 \mathrm{~cm}^{-1}$ (aliphatic C-H); $1697.36 \mathrm{~cm}^{-1}(\mathrm{C}=0)$ and 1612.49 ; 1570.06; $1496.76 \mathrm{~cm}^{-1}$ (aromatic $\mathrm{C}=\mathrm{C}$ ).
Analysis of ${ }^{1} \mathrm{H}-\mathrm{NMR}$ (acetone-d6, $500 \mathrm{MHz}$ ) showed presence of 9 protons. Doublet signal on $\delta_{\mathrm{H}} 6.2911 \mathrm{ppm}(d, 1 \mathrm{H}, J=10)$, and $\delta_{\mathrm{H}} 7.8600 \mathrm{ppm}$ $(d, 1 \mathrm{H}, J=10)$ showed correlation due to the signals and certain signal for unsubstitute coumarin in orto position on pyron ring (Murray et al., 1982). Methoxy group $\left(-\mathrm{OCH}_{3}\right)$ showed $\delta_{\mathrm{H}} 3.8773(s, 3 \mathrm{H})$ and $\delta_{\mathrm{H}} 3.8850(s, 3 \mathrm{H})$ signals.

Analysis of ${ }^{13} \mathrm{C}-\mathrm{NMR}$ (acetone-d6, $125 \mathrm{MHz}$ ) showed presence of 11 carbons. Signals on $\delta_{c}$ 56.5453 and 61.2191 ppm came from 2 methoxy groups $\left(-\mathrm{OCH}_{3}\right)$. Signal on $\delta_{\mathrm{C}} 160.7039 \mathrm{ppm}$ came from carbonyl group ( $\mathrm{C}=0$ ) (Pretsch et al., 2009). 
The ${ }^{1} \mathrm{H}-\mathrm{NMR}$ and ${ }^{13} \mathrm{C}-\mathrm{NMR}$ data of isolate 1 were founded to be identified as 8-hydroxy-6,7dimethoxy coumarin (fraxidin).

The structure of fraxidin showed on figure 3 was confirmed by comparison of 2D-NMR spectral data with earlier published literature of Fraxidin data (Rumzhum et al., 2012).<smiles>COc1cc2ccc(=O)oc2c(O)c1OC</smiles>

Figure 3. 8-hydroxy-6,7-dimethoxy coumarin

\section{CONCLUSION}

Ethyl acetate extract of Jatropha multifida L. had toxicity value with $\mathrm{LC}_{50} 3.69 \mu \mathrm{g} / \mathrm{mL}$ with BSLT assay. Isolate 1 was succesfull isolated and identified as 8-hydroxy-6,7-dimethoxy coumarin and not classified as toxic compound after BSLT assay.

\section{REFERENCES}

Das, B., Laxminarayana, K., Krishnaiah, M., Srinivas, Y. dan Raju, T. V. (2009): Multidione, A Novel Diterpenoid From Jatropha multifida, Tetrahedron Letters, 50 (34), 4885-4887.

Das, B., Ravikanth, B., Reddy, K. R., Thirupathi, P., Raju, T. V. dan Sridhar, B. (2008a): Diterpenoids from Jatropha multifida, Phytochemistry, 69 (14), 2639-2641.

Das, B., Reddy, K. R., Ravikanth, B., Raju, T. V., Sridhar, B., Khan, P. U. dan Rao, J. V. (2008b): Multifidone: A Novel Cytotoxic Lathyrane-Type Diterpene Having An Unusual Six-Membered A Ring From Jatropha multifida, Bioorganic \& medicinal chemistry letters, 19 (1), 77-79.

Depkes (2000): Parameter Standar Umum Ekstrak Tumbuhan Obat, Direktorat Jenderal Pengawasan Obat dan Makanan Direktorat
Pengawasan Obat Tradisional, Jakarta.

Farnsworth, N. (1966): Biological and Phytochemical Screening of Plants, Journal of Pharmaceutical Sciences, 55 (3), 243268.

Honasan, Y. (2012): Isolasi dan Uji Toksisitas Senyawa Toksik Golongan Terpenoid dan Fenol Daun Jarak Tintir (Jatropha multifida Linn.) dengan Metode Brine Shrimp Lethality Test (BSLT), Skripsi, Jurusan Farmasi Fakultas MIPA Universitas Jenderal Achmad Yani, Cimahi.

Hutapea, J. (2000): Inventaris Tanaman Obat Indonesia, Departemen Kesehatan RI dan Badan Penelitian dan Pengembangan Kesehatan, Jakarta.

Kanth, B. S., Kumar, A. S., Shinde, D. B., Babu, K. H., Raju, T. V., Kumar, C. G., Sujitha, P. dan Das, B. (2011): New Bioactive Macrocyclic Diterpenoids From Jatropha multifida, Bioorganic \& medicinal chemistry letters, 21 (22), 6808-6810.

McLaughlin dan Jerry, L. (1991): Crown Gall Tumours on Potato Discs and Brine Shrimp Lethality: Two Simples Bioassays for Higher Plant Screening and Fractination, Methods in Plant Biochemistry, 6, 8-10.

Meyer, B. N., Ferrigni, N. R., Putnam, J. E., Jacobsen, L. B., Nichols, D. E. dan McLaughlin, J. L. (1982): Brine Shrimp: A Convenient General Bioassay for Active Plant Constituents, Planta Medica, 45 (5), 3134.

Murray, R. D. H., Mendez, J. dan Brown, S. A. (1982): The Natural Coumarins: Occurrence, Chemistry, and Biochemistry, John Wright \& Sons, Bristol.

Pretsch, E., Buhlmann, P. dan Badertscher, M. (2009): Structure Determination of Organic Compounds, Springer, Berlin.

Rumzhum, N. N., Sohrab, M. H., Al-Mansur, M. A., Rahman, M. S., Hasan, C. M. dan Rashid, M. A. (2012): Secondary Metabolites from Jatropha podagrica Hook Journal of Physical Science, 23 (1), 29-37. 